\title{
Le PNR 67 «Fin de vie» éclaire les défis
}

\author{
Jean Martin \\ Dr med., membre de la rédaction
}

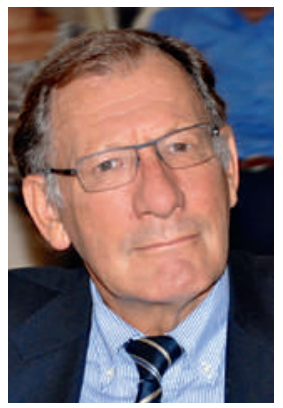

Le programme national de recherche 67 , «Fin de vie», a été lancé en 2011 avec un financement de 15 millions de francs pour cinq ans. Ont été sélectionnés les projets de 33 équipes. En 2016, ses responsables organisent cinq dialogues permettant de discuter les résultats obtenus. Le premier, "Mourir à la maison ou dans un EMS», a eu lieu à Berne le 19 février. On y a beaucoup parlé des proches aidants, de plus en plus sollicités pour prendre soin de leurs parents très âgés, une problématique qui croît rapidement en importance. Un travail sous la direction de B. Sottas, réalisé à Fribourg et en Valais, a étudié les situations critiques à cet égard. Ainsi, la durée des aides extérieures, professionnelles et autres, est

Il est impératif de mieux reconnaître le rôle des proches aidants et de mieux les soutenir.

d'un peu plus d'une heure par jour, alors que les proches aidants doivent être disponibles en permanence. Ces derniers ont de la peine à y voir clair: pour un patient, on a pu voir jusqu'à 32 intervenants, chacun pour une prestation particulière. D'où un très grand besoin de coordination. Parmi les conclusions de l'étude: les aidants sont souvent épuisés, au plan physique et psychique; ils (mais noter que ces aidants sont très majoritairement des femmes) ont le sentiment d'être abandonnés; ils peinent à se préparer au décès prévisible du patient, ont peur de ne pas faire juste ni assez et de ne plus avoir le contrôle, ni de la situation ni de leur propre vie.

Une étude lausannoise, sous la direction de M.-A. Berthod, s'est intéressée à la manière dont les aidants parviennent - ou pas - à concilier vie professionnelle et exigences du soutien à leur proche âgé. Les histoires recueillies éclairent les façons dont les gens s'adaptent. Pas surprenant d'observer que des aménagements sont rendus plus ou moins faciles - ou difficiles - selon la culture d'entreprise et la bonne volonté des supérieurs. Les auteurs de l'étude soulignent les besoins d'instauration de possibilités mieux structurées, si possible à large échelle, pour faciliter la disponibilité des proches aidants, y compris par une protection de l'emploi pour ceux qui diminuent leur temps de travail, ainsi que des soutiens organisationnels et matériels. Une plateforme nationale «Work and Care» sera lancée à ce propos en automne 2016. Là comme ailleurs, il y a une place pour des bénévoles, tout en sachant que l'engagement de ces derniers doit être fiable et adéquatement coordonné pour être utile.

La recherche présentée par A.-V. Dürst, S. Monod et coll. (Lausanne) a porté sur le désir de mourir dans des groupes de patients âgés: en service de réadaptation, $12,9 \%$ ont exprimé un tel désir; en EMS, ils étaient $21 \%$. A été évoquée la question du suicide assisté: les chiffres en Suisse montrent qu'il est plus fréquent chez les plus de 65 ans (et chez les plus de 75 ans, le nombre de suicides assistés est proche de celui des autres suicides) De manière intéressante, les auteurs estiment qu'il est possible d'évaluer adéquatement le désir de mourir chez des personnes avec un déficit cognitif modéré et même important [1].

Trois experts se sont exprimés sur les résultats présentés, dont la responsable de la stratégie à l'Office fédéral de la santé publique. Les questions sont complexes, c'est le moins qu'on puisse dire. D'abord il y a celle de la multiplicité des intervenants, qu'il faut chercher à simplifier, mais sans diminuer la qualité des prises en charge. Il est impératif de mieux reconnaître le rôle des proches aidants et de donner plus d'attention aux aides dont ils ont besoin: pour concilier leur engagement avec leur activité professionnelle, pour être parfois déchargés par d'autres, pour bénéficier d'informations et d'une certaine formation. En pratique, les responsabilités et tâches sont le fait de services privés ou publics. Dans le secteur public, la répartition des compétences diffère selon les cantons entre ce qui est du ressort des communes, voire des districts, et de l'Etat. On est habitué en Suisse à ces diversités, souvent solidement ancrées politiquement, mais il importe de faire en sorte que, partout et de manière équitable, nos concitoyens âgés et dépendants puissent bénéficier de prestations qualitativement et quantitativement adéquates. Clairement, les défis lancés par le «tsunami gris» du vieillissement sont de taille; il est bon que le PNR 67 mette le doigt sur des domaines et articulations qui demandent à être considérés en urgence.

\footnotetext{
Geronsions. Clinical Gerontologist. Posted 2015.
} 